А. А. С м е д о в, С. В. Т а и ло в,

К. А. По н о м р ев, А. Н. Ми ронихин

\title{
ОСОБЕННОСТИ ПОСТАНОВКИ И РЕШЕНИЯ
} ЗАДАЧ ОПТИМИЗАЦИИ СТРУКТУРЫ И СОСТАВА ПРОСТРАНСТВЕННО АРМИРОВАННЫХ УГЛЕРОД-УГЛЕРОДНЫХ И УГЛЕКЕРАМИЧЕСКИХ КОМПОЗИТНЫХ КОНСТРУКЦИЙ РАКЕТНОЙ ТЕХНИКИ

Приведень особенности выбора варьируемых параметров, формулировки критериев качества и отбора расчетньх и оптимизачионных алгоритмов для задач оптимизации структуры и состава пространственно армированных углерод-углеродных и углекерамических композитных конструкций ракетной техники. На конкретном примере показаны возможности оптимизации структуры и состава композиционного материала боковой поверхности неуправляемого спускаемого аппарата при поиске оптимального распределения плотностей и оптимальной толщины силицированного слоя.

\section{E-mail: asmerdov@mail.ru; Kirill.a.ponomarev@gmail.com}

Ключевые слова: оптимальное проектирование, композиционный материал, пространственное армирование, углерод-углеродный материал, углекерамика.

Задачи оптимального проектирования композитных структур широко освещаются в современной научно-технической литературе, и актуальность таких задач остается высокой на протяжении многих лет. С одной стороны, новые возможности, которые открываются перед проектантом композитных конструкций, требуют точного выбора большого числа варьируемых параметров. С другой - для современных композитов с их высокой степенью анизотропии резко возрастает опасность неблагоприятного сочетания свойств конструкции при недостаточно продуманном назначении ее параметров.

Абсолютное большинство работ в данной области, начиная с классических монографий [1-4] и заканчивая постоянно появляющимися статьями в специальных изданиях, посвящено многослойным структурам, содержащим однонаправленные или перекрестно армированные слои волокнистого материала. Значительно меньше внимания уделяется вопросам оптимизации пространственно армированных композитов, в частности композитов с углеродной или керамической матрицей. Это связано прежде всего со сложностью технологической реализации подобных конструкций, дороговизной и значительным временем (а следовательно, и ограниченным объемом) их отработки, когда отработанная технология практически не имеет альтернативы.

Вместе с тем, появляются новые возможности улучшения свойств углерод-углеродных и углекерамических композиционных материалов 
(УУКМ и УККМ) и изделий из них. Это обусловливает новый взгляд на проблемы оптимального проектирования таких конструкций.

Пространственно армированные структуры из УУКМ и УККМ сегодня достаточно широко применяются в изделиях ракетной техники. Основная сфера их применения - теплонапряженные высоконагруженные узлы и детали ракетных конструкций. Таковы сопловые вкладыши ракетных двигателей и носовые вставки блоков, входящих с высокими скоростями в плотные слои атмосферы. Типичные примеры конструкций подобного рода показаны на рис. 1.

Говоря об особенностях постановки задач оптимального проектирования пространственно армированных композитных конструкций ракетной техники, прежде всего следует отметить ограниченность классов структур, параметры которых подлежат выбору. Так, на сегодняшний день для армирования осесимметричных конструкций ракетно-космической техники фактически используются всего две схемы (рис. 2): армирование 3D, когда оси армирующих элементов направлены по трем осям декартовой системы координат, и армирование 4DL, когда одно из направлений армирующих элементов совпадает с осью осесимметричной конструкции, а три остальных направления образуют квазиизотропную структуру [5] в плоскости, перпендикулярной этой оси, будучи размещены по схеме $\left[0 /+60^{\circ} /-60^{\circ}\right]$. Разумеется, можно придумать и другие варианты пространственного расположения армирующих элементов [6], однако эти варианты
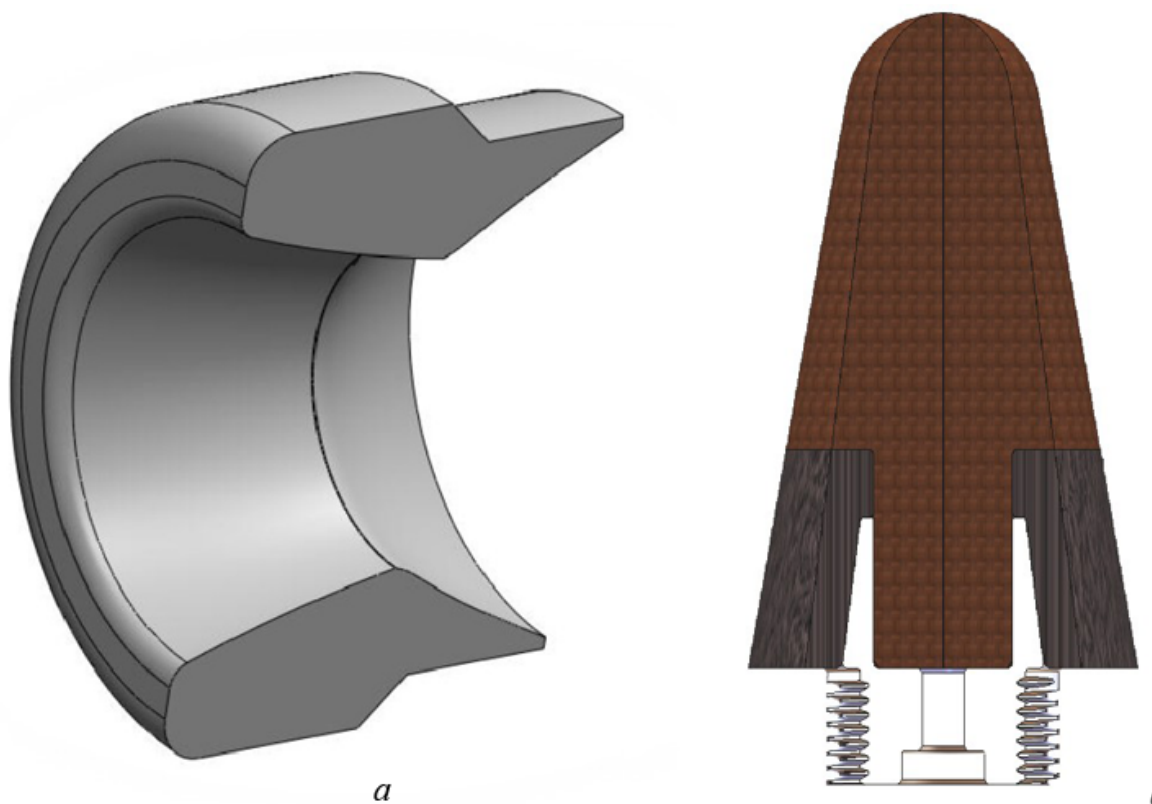

$\sigma$

Рис. 1. Примеры композитных пространственно армированных конструкций: сопловой вкладыш ракетного двигателя $(a)$ и носовая вставка блока, входящего с высокой скоростью в плотные слои атмосферы (б) 


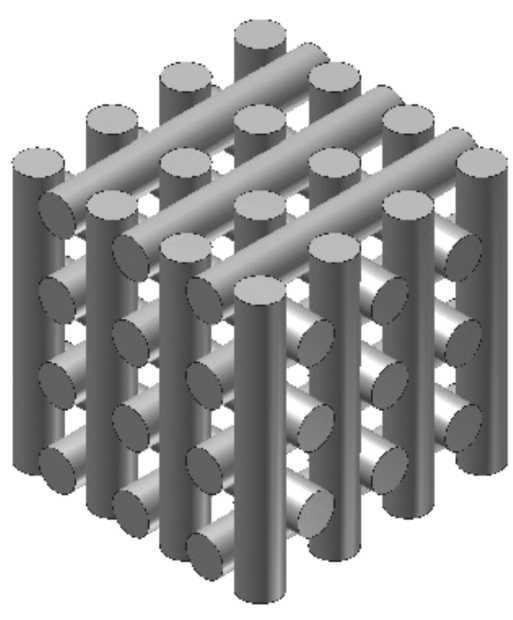

$a$

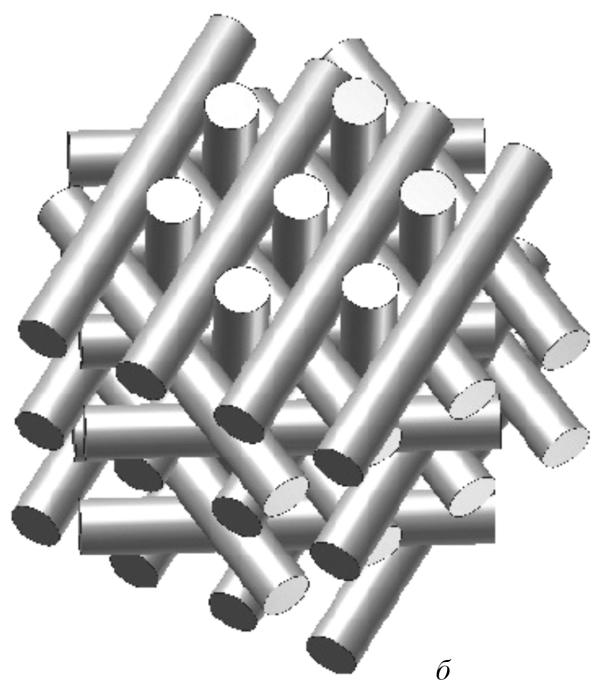

$\sigma$

Рис. 2. Основные схемы армирования ракетных конструкций из УУКМ и УККМ:

$a$ - армирование $3 \mathrm{D} ; \sigma$ - армирование 4D

чрезвычайно трудно реализовать, а рациональные соображения в их пользу отсутствуют.

Таким образом, действия расчетчика при проектировании новой композитной конструкции в данной области фактически сводятся к параллельной оптимизации двух указанных схем с последующим выбором наилучшей из них по совокупности анализируемых характеристик.

Следующая особенность связана с весьма ограниченными возможностями варьирования структурных параметров композита. Традиционная постановка задач оптимизации слоистых композитов [7] относит к числу варьируемых параметров относительные толщины и углы ориентации отдельных слоев; для композитных конструкций обычно добавляются параметры, характеризующие чередование слоев в многослойном пакете [2]. Как правило, свойства проектируемой конструкции сильно зависят от таких варьируемых параметров: так, для простейших перекрестно армированных и ортогонально армированных структур из углепластика, изменяя только структурные параметры, можно добиться изменения жесткостных и прочностных характеристик на два порядка, коэффициентов теплопроводности - более, чем на порядок, а коэффициенты линейного термического расширения могут отличаться даже знаком [7].

При изменении параметров армирования УУКМ и УККМ (в пределах выбранной схемы армирования) результаты далеко не столь очевидны. Фактически единственным варьируемым параметром является диаметр армирующих элементов - стержней. Эти стержни формуются отдельно и составляют в дальнейшем каркас, на который тем 
или иным путем осаждается углеродная матрица или матрица более сложного состава [8]. Упаковка стержней в пределах выбранной схемы выбирается максимально плотной; таким образом, диаметр стержней определяет размер ячейки композитной структуры. В свою очередь, свойства композитной структуры довольно сложным образом зависят от этого размера.

Следующая (и, возможно, наиболее важная) особенность рассматриваемых задач оптимизации - возможность варьирования не только структуры, но и состава проектируемого материала. Как правило, при оптимизации многослойных структур наименьшей, далее неделимой, единицей считается однонаправленный или перекрестно армированный монослой $[5,7]$. Свойства такого монослоя в естественной системе координат заданы и не изменяются в процессе оптимизации. Это обеспечивается технологией производства многослойных волокнистых композитов, когда параметры монослоя, определяемые прежде всего характеристиками волокон и матрицы и соотношением их в объеме материала, отрабатываются заранее и не подлежат изменению. В данном случае дело обстоит иначе, поскольку матрица формируется на углеродном каркасе непосредственно в процессе создания композитной конструкции, а технологические процессы формования этой матрицы за последние годы были значительно усложнены и расширены, так что стали возможными постановки задач оптимизации состава композитного материала или соответствующей конструкции.

Наиболее интересная особенность варьирования состава пространственно армированных УУКМ и УККМ - возможность создания неоднородных по объему материалов, требования к которым могут быть сформулированы в каждой конкретной задаче исходя из требований к жизненному циклу конструкции, для которой создается этот материал. Перспективным представляется использование УУКМ и УККМ, обладающих переменными по толщине характеристиками. При этом наружные, более плотные слои материала обеспечивают необходимую эрозионную стойкость и конструкционную прочность, а внутренние слои с малой плотностью предназначены выполнять роль тепловой изоляции, которая обеспечивает необходимые температурные режимы внутри конструкции, для надежной работы управляющих приборов.

Помимо изменения плотности, при рациональном проектировании элементов из пространственно армированных углеродных композитов могут варьироваться параметры силицирования углеродной матрицы, причем размер силицированной зоны также подлежит оптимизации. Кроме того, возможное изменение состава пространственно армируемого композита может включать в себя также использование присадок тугоплавких материалов.

Таким образом, варьируемые параметры в рассматриваемых задачах отличаются многообразием. 
Вместе с тем, требования к свойствам проектируемых конструкций также могут сильно отличаться от традиционных условий, предъявляемых к свойствам многослойных структур. Так, для вышеупомянутых теплонапряженных элементов конструкций ракетной техники могут ставиться задачи поиска оптимального распределения свойств по толщине композитной детали, наилучшим образом соответствующего изменению во времени термосиловых нагрузок на эту деталь и требованиям к сохранению ее свойств при данных нагрузках.

Целевые функции и ограничения в рассматриваемых задачах оптимизаций обычно весьма сложны. Они могут включать в себя характеристики процессов, протекающих во времени. Так, для конструкций со значительным уносом массы может быть исследовано последовательное изменение формы и размеров теплонапряженного элемента конструкции и поставлены требования к его конфигурации в некоторые заданные моменты времени.

При наличии столь сложных целевых функций и ограничений число рассматриваемых проектов при поиске оптимальной конструкции должно быть сильно ограничено. В результате возникают особые требования к оптимизационным алгоритмам. Однако весьма сложные и неочевидные зависимости критериев качества от варьируемых параметров требуют использования простых и надежных алгоритмов поиска, таких как глобальный перебор по сетке [9]. При этом разбивка сетки по необходимости должна быть достаточно редкой. Для повышения точности определения оптимальных параметров процесс поиска может быть проведен в несколько этапов с постепенным сужением сетки.

В качестве примера рассматривается задача оптимизации композиционного материала боковой поверхности неуправляемого спускаемого аппарата при следующих требованиях:

- целевая функция - минимальное значение температуры на внутренней границе композитной стенки к концу работы конструкции;

- ограничение 1 - обеспечение прочности материала конструкции во все время ее работы;

- ограничение 2 - обеспечение заданного уноса материала с наружной поверхности, не превышающей толщину силицированного внешнего слоя конструкции.

Суть решаемой задачи состоит в поиске оптимального распределения плотностей (т.е. степени насыщения углеродом УУКМ) и оптимальной толщины силицированного слоя (т.е. оптимального соотношения между УУКМ и УККМ) для данной конструкции.

Исходными данными для решения этой задачи являются:

- толщина стенки $H$;

- описание программы входа изделия в атмосферу, определяющей последовательность приложения термосиловых нагрузок к дан- 
ной конструкции (содержит запись о числе точек траектории и их перечисление в формате: момент времени, скорость, высота полета);

- таблица зависимости теплоемкости материала от температуры (для углеродных материалов теплоемкость не зависит от структуры материала и его плотности);

- таблицы распределения теплопроводности, температурных коэффициентов линейного расширения, характеристик жесткости и прочности композита в зависимости от плотности материала и температуры;

- разделение стенки конструкции на отдельные слои, каждый из которых имеет свои характеристики, обеспечиваемые структурой и составом данного слоя, причем внешний из слоев силицирован и представляет собой УККМ, а прочие - УУКМ с различной степенью насыщения, а следовательно, с различной плотностью и прочими теплофизическими и термомеханическими характеристиками;

- задание предельных условий прочности каждого слоя в зависимости от его структуры и состава, а также задание требований к уносу массы внешнего слоя, определяющее толщину унесенного слоя к концу программы входа в атмосферу.

\section{Постановка задачи оптимизации и оптимизационный алго-} ритм. Стенка конструкции разделяется на три слоя:

- поверхностный слой, плотность которого максимальна для данной технологии, а толщина $h_{1}$ варьируется в заданных пределах и с заданным шагом;

- внутренний слой, плотность которого, как и его толщина $h_{3}$, является варьируемым параметром;

- средний слой, толщина которого $h_{2}$ равна разности между толщиной стенки и суммой толщин внутреннего и внешнего слоев (при варьировании должно соблюдаться условие $h_{1}+h_{3} \leq H$, которое для гарантированного обеспечения работы алгоритма принимается в виде $\left.h_{1 \max }+h_{3 \max } \leq H\right)$.

На рис. 3 показаны крайние варианты распределения толщин от внешнего до внутреннего слоя, предусмотренные оптимизационным алгоритмом, в котором толщина промежуточного слоя $h_{2}$ не является варьируемым параметром, а определяется диапазонами варьирования параметров $h_{1}$ и $h_{3}$. В пределах поверхностного слоя отдельно выделяется силицированный слой, толщина которого $h_{4}$ (рис.4) также изменяется в заданных пределах и с заданным шагом.

Итого имеется 4 варьируемых параметра: толщины поверхностного $\left(h_{1}\right)$, внутреннего $\left(h_{3}\right)$ и силицированного $\left(h_{4}\right)$ слоев, а также плотность внутреннего слоя.

Поиск оптимального проекта осуществляется с помощью глобального перебора по сетке. Разбивку выполняют таким образом, чтобы 
Плотность, кг/ $\mathbf{M}^{3}$

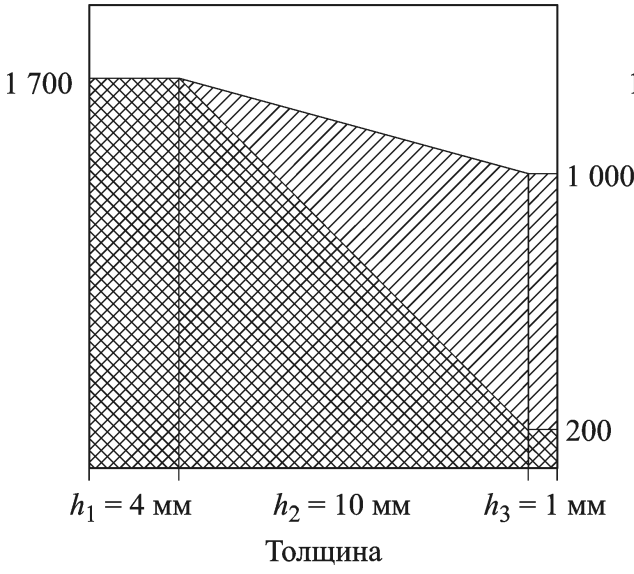

a

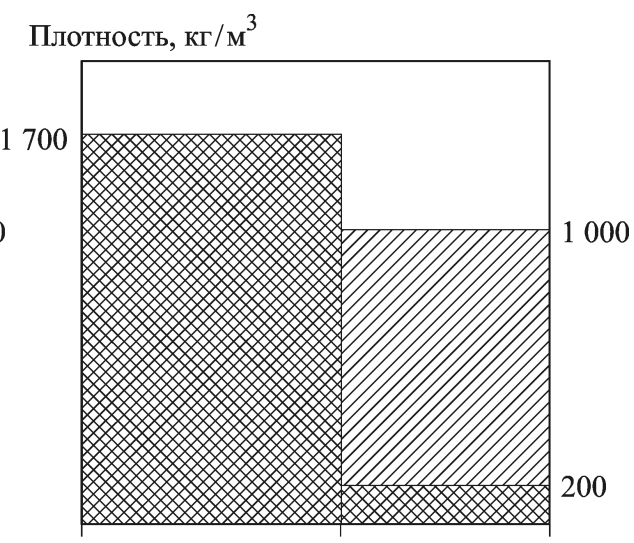

$$
h_{1}=8 \mathrm{MM} \quad h_{3}=7 \mathrm{MM}
$$

Толщина

6

Рис. 3. Крайние варианты распределения толщин внутреннего, внешнего и промежуточного слоев, предусмотренные оптимизационным алгоритмом

число обращений к процедуре вычисления свойств текущего варианта конструкции не превышало 300.

Тепловой расчет элемента конструкции проводится путем решения линейного дифференциального уравнения теплопроводности в одномерной цилиндрической постановке [10, 11$]$.

Решение задачи методом конечных разностей ведется по неявной схеме, что обеспечивает абсолютную устойчивость решения, с переменным шагом по времени. Шаг по времени выбирают, исходя из характерных линейных размеров конструкции, свойств материала, а также темпов нагрева. В целях ускорения расчета предусмотрены динамические уменьшение и увеличение временного шага.

Решение разрешающей системы линейных алгебраических уравнений ведется методом прогонки, поскольку ее матрица является трехдиагональной.

В качестве граничных условий принимаются: граничное условие второго рода на внешней стенке, где вычисление теплового потока осуществляется в аэродинамическом расчете, и граничное условие второго рода на внутренней стенке - тепловой поток равен нулю.

Каждый шаг решения состоит из нескольких этапов:

- расчет координатной сетки для каждого из заданных слоев стенки;

- интерполяция плотностей материала и температур участков в узлах полученной сетки;

- интерполяция теплофизических свойств материала в узлах сетки исходя из полученных значений плотностей и температур;

- определение параметров текущей точки условной траектории; 
- определение параметров атмосферы и свойств набегающего потока для текущей точки траектории;

- аэродинамический расчет с учетом скорости и свойств атмосферы в текущей точке траектории, а также температурного поля стенки;

- заполнение матрицы коэффициентов и вектора свободных членов разрешающей системы линейных алгебраических уравнений;

- решение полученной системы методом прогонки;

- проверка условий по шагу по времени с возможной его корректировкой и повторным расчетом;

- расчет уноса материала с внешней поверхности конструкции в соответствии с распределением температурного поля по стенке и обновление геометрических параметров расчетной модели.

После каждого шага расчета осуществляются передача его результатов в процедуру прочностного расчета и их вывод во внешние файлы в текстовом формате.

Аэродинамический расчет проводится для определения теплового потока на внешней стенке [12]. При проведении аэродинамического расчета используется заданная условная траектория. На каждом шаге перед проведением основного расчета определяются параметры текущей точки траектории, а затем по известным высоте и скорости изделия - параметры атмосферы и свойства набегающего потока в данной точке.

Каждый шаг решения состоит из нескольких этапов:

- формирование условного профиля поверхности изделия с учетом удаленности рассматриваемой зоны;

- расчет параметров набегающего потока для заданных параметров точки траектории;

- определение точки отрыва набегающего потока;

- определение параметров ударной волны;

- расчет распределения давления по условному профилю поверхности;

- определение параметров ламинарного и турбулентного потоков вдоль условного профиля;

- расчет теплового потока к поверхности вдоль условного профиЛя;

- выделение значений давления и теплового потока для рассматриваемой точки.

После аэродинамического расчета по полученным значениям давления на поверхности и температуры стенки рассчитывают скорость уноса материала [13]. Данные о тепловом потоке, внешнем давлении и скорости уноса материала с поверхности в текущий момент передаются для продолжения расчета. 
Механический расчет - это расчет напряженно-деформированного состояния участка конструкции путем решения уравнения теории упругости в квазиодномерной цилиндрической постановке методом начальных параметров [14].

При расчете учитываются значения температуры, геометрические параметры конструкции, (а также сетка), полученные в ходе ее теплового расчета. Принимаются следующие допущения:

- напряженно-деформированное состояние слабо изменяется вдоль оси симметрии;

- в конструкции отсутствуют изгибные деформации;

- для материала конструкции выполняется закон Гука во всем интервале деформаций.

В качестве граничных условий принимаются:

- значение давления на внешней стенке, полученное из аэродинамического расчета;

- значение давления на внутренней стенке, равное атмосферному.

При формировании заключения о сохранении конструкцией прочности используется критерий наибольших напряжений.

Процесс решения состоит из следующих этапов:

- интерполяция механических свойств материала в узлах сетки исходя из существующих значений плотностей и температур;

- предсказание значения осевой деформации;

- формирование матриц перехода через участки конструкции;

- осуществление метода начальных параметров;

- получение значений перемещений, деформаций и напряжений в узлах сетки;

- проверка условия по осевому усилию, при необходимости корректировка значения осевой деформации и повтор расчета;

- проверка условия прочности конструкции для всех узлов сетки, получение заключения о прочности конструкции.

Результаты расчета. Решена конкретная оптимизационная задача, получены значения варьируемых параметров (рис. 4). Результаты показаны на фоне крайних значений варьируемых параметров. (Толстая сплошная линия - распределение плотности для оптимального проекта; штриховая - толщина силицированного слоя.) При этом температура на внутренней стенке составила $347 \mathrm{~K}$; все ограничения по прочности и величине уноса выполнены.

Видно, что минимальные значения плотности (см. рис. 3) оказываются недоступны из-за нарушения ограничений по прочности; представленный проект является результатом компромисса между требованиями минимальной температуры на внутренней стенке и условиями прочности конструкции. 


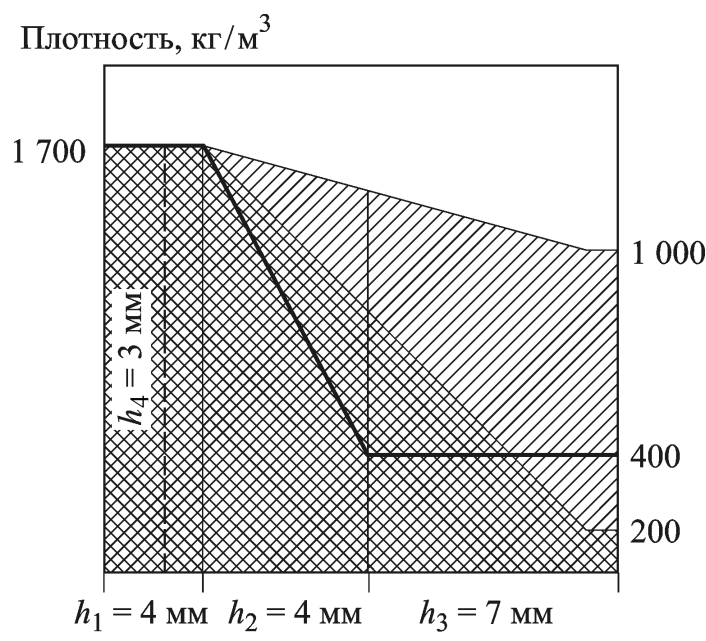

Толщина

\section{Рис. 4. Результаты решения задачи оптимизации}

Представленный пример иллюстрирует сложность оптимизационных задач для конструкций данного класса и особенности представления варьируемых параметров, критериев качества и алгоритмов решения.

В заключение следует отметить, что значительный интерес представляет анализ данного типа задач в рамках векторной оптимизации $[9,15]$. Эти проблемы могут быть рассмотрены по мере накопления опыта в постановках и решении задач оптимизации структуры и состава пространственно армированных УУКМ и УККМ ракетной техники.

\section{СПИСОК ЛИТЕРАТУРЫ}

1. Б а н и ч у к Н. В., К о б е л е в В. В., Р и к а р д с Р. Б. Оптимизация элементов конструкций из композиционных материалов. - М.: Машиностроение, 1988.$224 \mathrm{c}$.

2. Т е т е р с Г. А., Р и к а рд с Р. Б., Н а р у с б е р г В. Л. Оптимизация оболочек из слоистых композитов. - Рига: Зинатне, 1978. - 240 с.

3. $\mathrm{C}$ h o u T-W. Microstructural design of fiber composites. - Cambridge: Cambridge University Press, 1992. - 569 p.

4. A s h b e e K. H. G. Fundamental principles of fiber reinforced composites. Lancaster (USA): Technomic Publishing Co., Inc., 1993. - 424 p.

5. А л ф у т о в Н. А., 3 и н о в ь е в П. А., По п о в Б. Г. Расчет многослойных пластин и оболочек из композиционных материалов. - М.: Машиностроение, 1984. - 264 c.

6. Ж и г у н И. Г. Свойства пространственно-армированных композитов // Композиционные материалы: Справочник / Под ред. В.В. Васильева, Ю.М. Тарнопольского. - М.: Машиностроение, 1990. - С. 267-301.

7. 3 и н о в ь е в П. А., С м ерд в в А. А. Оптимальное проектирование композитных материалов. - М.: Изд-во МГТУ им. Н.Э. Баумана, 2006. - 103 с.

8. Щ у р и к А. Г. Искусственные углеродные материалы. - Пермь, 2009. - 342 с.

9. С м е р д о в А. А. Основы оптимального проектирования композитных конструкций. - М.: Изд-во МГТУ им. Н.Э. Баумана, 2006. - 88 с. 
10. К а р с л о у Г., Е г е р Д. Теплопроводность твердых тел. - М.: Наука, 1964. $488 \mathrm{c}$.

11. П о т т е р Д. Вычислительные методы в физике. - М.: Мир, 1975. - 392 с.

12. А в д у е в с к и й В. С., Гали це й ск ий Б. М., Гле бов Г. А. Основы теплопередачи в авиационной и ракетно-космической технике. - М.: Машиностроение, 1992. - 528 с.

13. Ш л е н с к и й О. Ф., Ш а ш ко в А. Г., А к с н о в Л. Н. Теплофизика разлагающихся материалов. - М.: Энергоатомиздат, 1985. - 145 с.

14. Б а л а б у х Л. И., А л фу т о в Н. А., У с ю к и н В. И. Строительная механика ракет. - М.: Высш. шк., 1984. - 392 с.

15. Б о р и с о в В. И. Проблемы векторной оптимизации // Исследование операций. - М.: Наука, 1972. - С. 72-91.

Статья поступила в редакцию 15.05.2012 